\title{
The Alzheimer's Prevention Initiative Generation Program: Evaluating CNP520 Efficacy in the Prevention of Alzheimer's Disease
}

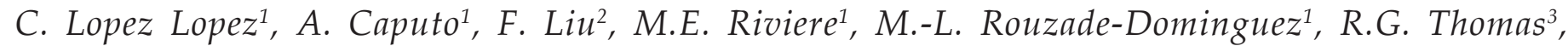 \\ J.B. Langbaum ${ }^{5}$, R. Lenz $z^{4}$,E.M. Reiman ${ }^{5}$, A. Graf ${ }^{1}$, P.N. Tariot ${ }^{1}$
}

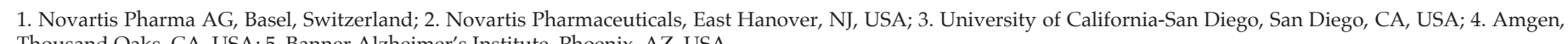
Thousand Oaks, CA, USA; 5. Banner Alzheimer's Institute, Phoenix, AZ, USA

Corresponding Author: Cristina Lopez Lopez, MD, PhD, Neuroscience Development Unit, Global Drug Development, Novartis Campus, Fabrikstrasse 12-4.03.38, 4056 Basel, Switzerland, Phone: +41 79865 9366, Email: cristina.lopez_lopez@novartis.com

\begin{abstract}
Alzheimer's disease pathology begins decades before the onset of clinical symptoms. This provides an opportunity for interventional clinical trials to potentially delay or prevent the onset of cognitive impairment or dementia. CNP520 (a beta-site-amyloid precursor protein-cleaving enzyme inhibitor) is in clinical development for the treatment of preclinical Alzheimer's disease under the Alzheimer's Prevention Initiative Generation Program. The Alzheimer's Prevention Initiative is a public-private partnership intended to accelerate the evaluation of Alzheimer's disease prevention therapies. The Generation Program comprises two pivotal phase II/III studies with similar designs to assess the efficacy and safety of investigational treatments in a cognitively unimpaired population at increased risk for developing Alzheimer's disease based on age and apolipoprotein E (APOE) genotype (i.e., presence of the APOE $\varepsilon 4$ allele). The program has been designed to maximize benefit to Alzheimer's disease research. Generation Study 1 (NCT02565511) and Generation Study 2 (NCT03131453) are currently enrolling; their key features are presented here.
\end{abstract}

Key words: Alzheimer's disease, BACE inhibitor, generation program.

\section{Introduction}

A lzheimer's disease (AD) is the most common degenerative brain disease, affecting approximately $10 \%$ of the population over 65 years of age (1). AD is characterized clinically by the presence of short-term memory loss, impaired judgment and problem solving, changes in mood and behavior, and eventual functional impairment, resulting in significant familial, societal and economic burden. Recent reports on the economic implications of AD in the US alone estimate that by 2050, 13.5 million people will have clinical AD dementia, resulting in Medicare costs of $\$ 627$ billion dollars (1). A treatment that could delay the onset of $\mathrm{AD}$ would have a tremendous public health benefit; postponing the onset of AD symptoms by five years has the potential to reduce the prevalence of $\mathrm{AD}$ by nearly $50 \%$ (2), and in turn reduce the economic burden dramatically.

Substantial evidence from genetic at-risk groups and otherwise cognitively unimpaired individuals suggests that progressive AD biomarker changes begin decades before mild cognitive impairment (MCI) or dementia. One critical, detectable change is the accumulation of pathological amyloid-beta $(\mathrm{A} \beta)$ species in the brain, beginning more than two decades before the onset of cognitive impairment.

This long preclinical phase of AD provides a critical window for AD prevention strategies. However, the development of AD treatments in this early disease stage requires novel scientific approaches: because of the unprecedented complexity of these study designs, duration of treatments, regulatory approval paths, ethical considerations, and required financial investments, this research may be better supported through public-private partnerships. The studies discussed here (collectively, The Generation Program) are supported by a partnership among Novartis, the Banner Alzheimer's Institute, and Amgen to bring collective resources together for the development and potential commercialization of a new prevention treatment for AD.

The Generation Program is one component of the Alzheimer's Prevention Initiative (API), a public-private partnership that was created to evaluate promising investigational prevention treatments in cognitively unimpaired people who are at the highest risk for the onset of clinical symptoms of AD (3). The API has several important goals that go beyond those of usual clinical trials, including: to provide a better test of the amyloid hypothesis than trials at later stages of $\mathrm{AD}$; to determine the extent to which treatment affects brain imaging and other biomarkers, predicting clinical benefit; to establish $\mathrm{AD}$ prevention registries needed to support recruitment into preclinical AD trials; to share data and samples with the scientific community; and to give healthy individuals at high risk for $\mathrm{AD}$ access to promising investigational treatments. In concordance with these goals, the Generation Program has a theragnostic biomarker development effort with the specific aim to 
correlate biomarkers for AD with therapeutic efficacy. This will help provide the evidence needed to qualify biomarker endpoints as potential surrogates for use in future prevention trials and could also provide a rationale to extend the evaluation of new therapies to amyloid negative subjects with genetic risk factors for AD. To support research in the AD scientific community, the baseline and post-baseline data from these studies will be shared. This resource will represent the largest data repository in subjects at the highest risk to develop symptoms of late onset $\mathrm{AD}$ based on their apolipoprotein $\mathrm{E}$ (APOE) genotype (i.e., presence of the APOE $\varepsilon 4$ allele) and the presence of underlying brain amyloid pathology.

\section{Risk factors for progression to AD}

Pathologically, AD is characterized by the accumulation of extracellular $\mathrm{A} \beta$ oligomers, plaques, and hyperphosphorylation of tau protein that is aggregated in intraneuronal neurofibrillary tangles. A causal role for aggregated and deposited forms of $\mathrm{A} \beta$ is supported by a vast body of histopathologic, genetic and biomarker studies.

APOE4 is the major genetic risk factor for developing Alzheimer's disease at older ages. Each additional copy of the APOE4 in a person's genotype is associated with a higher risk of $\mathrm{AD}$ and a younger age at dementia onset (Table1) (4-6). According to available prospective cohort data, cognitively unimpaired individuals, 60-75 years of age, who are APOE4 homozygous carriers (HMs) have a $30-55 \%$ risk of developing $\mathrm{MCI}$ or dementia due to $\mathrm{AD}$ by age 85 , and APOE4 heterozygote (HTs) carriers have a $20-25 \%$ risk (4).

The presence of APOE4 has been associated with reduced $A \beta$ clearance, increased $A \beta$ accumulation, increased $A \beta$-induced neurotoxicity, inflammation, reduced energy metabolism, impairment in mitochondrial function, and other processes relevant to $\mathrm{AD}$ risk $(6,7)$. APOE4 carriers have greater fibrillar amyloid deposition than age-matched non-carriers and accelerated age-dependent cognitive decline; further, the amount of amyloid deposition in preclinical AD individuals and rate of cognitive decline are directly associated with APOE4 gene dose (8). As previously noted, a recent longitudinal survival study has illustrated the age dependent effects of APOE4 in cognitively normal individuals who go on to develop clinical AD (4). APOE4 testing may help to identify people predisposed to progress clinically and can serve as a source of enrichment for clinical trials through a non-invasive and inexpensive test. Longitudinal data from prospective cohort studies are not yet available to accurately determine risk estimates for developing $\mathrm{MCI}$ or dementia due to AD in cognitively unimpaired APOE4 HTs with elevated brain amyloid.

\section{Current $\mathrm{A} \beta$ development strategies}

Given the compelling genetic and pathophysiological support for a causal association between $\mathrm{A} \beta$ and $\mathrm{AD}$, an effective treatment that targets $A \beta$ could have a profound impact on public health. Various investigational treatments targeting $A \beta$ are in late clinical development; however, several treatments have failed to meet their primary endpoints in cognition and activities of daily living. One widely held hypothesis for why these trials have failed is that the intervention occurred too late in the course of the disease. The vast majority of the failed trials enrolled subjects with mild-to-moderate $\mathrm{AD}$ - the amyloid pathology, the extensive appearance of tau (neurofibrillary) pathology and the irreversible synaptic or neuronal loss may have been too advanced for treatments to have any measureable impact. The development of drugs targeting amyloid focuses increasingly on the newly-defined earlier stage of $\mathrm{AD}$, i.e. $\mathrm{MCI}$ and preclinical $\mathrm{AD}$, which reflects current evidence that measurable changes in brain biomarkers may occur years before symptoms appear (1). We predict that the benefits of a disease-modifying therapy targeting $A \beta$ will be greatest during these early stages.

\section{The Generation Program}

The Generation Program comprises two pivotal studies, Generation Study 1 (NCT02565511) and Generation Study 2 (NCT03131453). The program is designed to provide efficacy, safety and tolerability data for two investigational $A \beta$-targeting drugs compared with matching placebo. CAD106 is a second generation, active $A \beta$ immunotherapy expected to enhance the clearance of $\mathrm{A} \beta$. CAD106 is tested in Generation study 1. CNP520, an orally-active inhibitor of beta-site amyloid precursor protein (APP) cleaving enzyme (BACE) that is involved in APP processing, has approximately 3-fold selectivity for BACE-1 over BACE-2 and no relevant off-target binding or activity.Following single and multiple dose administration of CNP520 in animals and humans, $A \beta$ concentrations in cerebrospinal fluid were reduced by up to $95 \%$ compared with baseline, and results of toxicology studies have not raised major safety concerns (Novartis, data on file). CNP520 is included in both Generation Study 1 (50 mg dose vs placebo) and Generation Study 2 ( 15 and $50 \mathrm{mg}$ vs placebo).

These clinical trials are recruiting cognitively unimpaired people who, on the basis of their age (60-75 years), genetics (carriage of APOE4) and presence of elevated amyloid (only in APOE4 HTs) are at particularly high risk of developing symptoms of AD (study design in Figure 1). Most of these people are considered to be in the preclinical stage of $\mathrm{AD}$, i.e., they are at particularly elevated risk of developing $\mathrm{AD}$ dementia but do not have overt symptoms and do not yet meet criteria for 


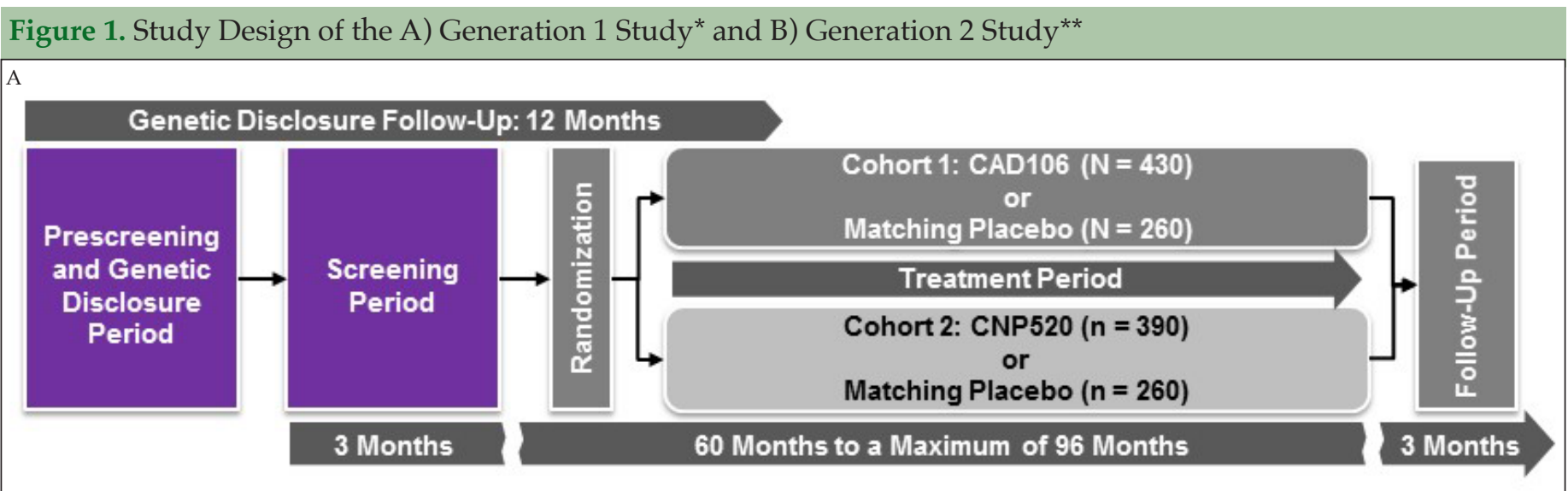

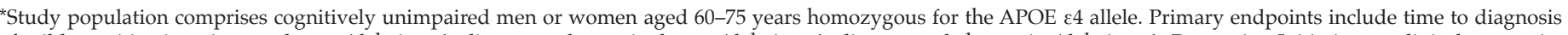

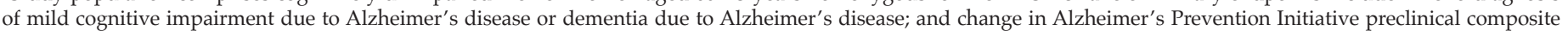
cognitive test score. Estimated study completion date: 2023. APOE, apolipoprotein E

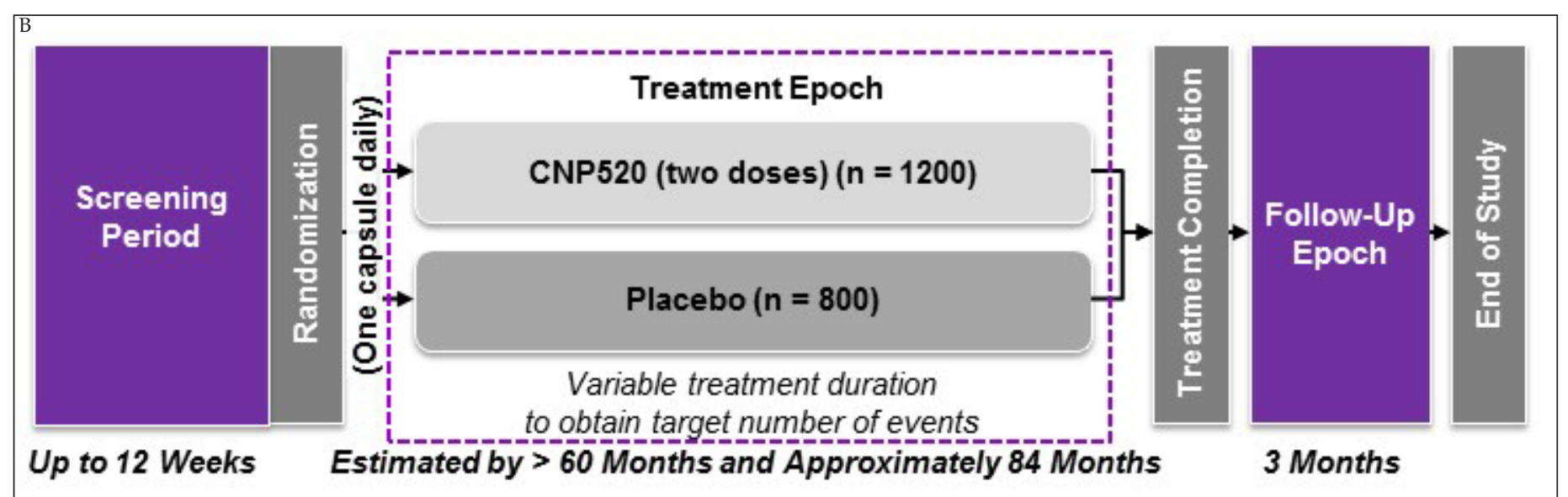

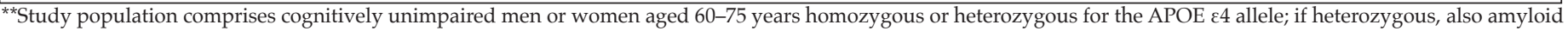

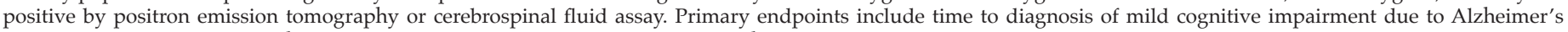

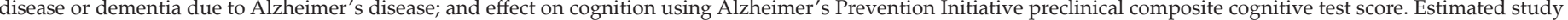
completion date: 2024. APOE, apolipoprotein E

approximately 1,300 cognitively healthy older adults, ages 60-75 years, who are APOE4 HMs, and Generation Study 2 will enroll 2,000 APOE4 carriers (APOE4 HMs or HTs; HTs must also have elevated brain amyloid load as assessed by either amyloid positron emission tomography imaging or cerebrospinal fluid assay). Participants will receive disclosure of their risk estimates to develop clinical symptoms of AD based on their genotype. Individuals are excluded from the studies if they have current neurological conditions; severe, progressive, or unstable disease that may interfere with the study assessments; brain magnetic resonance imaging findings that could lead to cognitive decline; or history of malignancy.

Both studies employ the same two primary outcomes: time-to-event (TTE) with event defined as diagnosis of MCI due to AD and/or dementia due to AD, and the API preclinical composite cognitive (APCC) test battery. The APCC battery was developed as a sensitive tool to detect and track cognitive decline in individuals at risk for progression to clinical stages of AD (9).

The Clinical Dementia Rating Sum of Boxes (CDR$\mathrm{SOB})$, a global measure widely used in clinical research in AD; Repeatable Battery for the Assessment of Neuropsychological Status (RBANS) total score, a clinical tool used to assess the neuropsychological status; and Everyday Cognition Scale (ECog), a measurement of daily function and subjective/study partner memory concerns will be included as secondary endpoints. These secondary endpoints are intended to thoroughly investigate potential drug effects and further assess the clinical relevance of treatment effects.

Based on the mechanism of CNP520 and CAD106, no short-term benefit is expected, particularly in this preclinical stage. It is expected that if the investigational drug delays the underlying pathological or pathophysiological disease processes, clinical benefit will emerge only gradually over time. As discussed in the draft CHMP Guideline (10), prevention trials require long treatment durations, typically at least five years. In these trials, participants will be treated for at least 60 months (five years) up to an expected maximum of 84 months (seven years). The minimum treatment duration of 60 months was chosen based on the likelihood of detecting (1) a sufficient number of events and (2) sufficient cognitive decline as measured by APCC test score in 
Table 1. Age and Genetics for At-Risk Alzheimer's Disease Population

\begin{tabular}{|l|c|c|c|c|}
\hline & $\begin{array}{c}\text { Percentage of General } \\
\text { Population (12) }\end{array}$ & $\begin{array}{c}\text { Lifetime MCI/AD Risk } \\
\text { by 85 Years (13) }\end{array}$ & $\begin{array}{c}\text { Percentage of AD } \\
\text { Population* (14) }\end{array}$ & $\begin{array}{c}\text { Age of Dementia } \\
\text { Diagnosis (Years) (6) }\end{array}$ \\
\hline APOE4 non-carriers & $73 \%$ & $10 \%-15 \%$ & $51 \%$ & 84 \\
\hline APOE4 heterozygote & $24 \%$ & $20 \%-25 \%$ & $39 \%$ & 76 \\
\hline APOE4 homozygote & $3 \%$ & $30 \%-55 \%$ & $10 \%$ & 68 \\
\hline
\end{tabular}

*Percentage of dementia due to Alzheimer's disease dementia population who were clinically characterized, not verified by neuropathology. AD, Alzheimer's disease; $\mathrm{APOE}$, apolipoprotein E; APOE4, $\mathrm{APOE} \varepsilon 4$ allele; $\mathrm{MCI}$, mild cognitive impairment.

the placebo arm to allow the detection of clinically meaningful treatment effects.

Recruitment is a key challenge as it requires screening of a cognitively unimpaired population willing to undergo genetic testing and risk disclosure. We estimate that investigators will need to screen approximately 100,000 people. This is a global program, including more than 20 countries and approximately 200 sites, which will recruit participants using a variety of approaches. For example, in order to support recruitment of US-based participants into these (and other studies), the Banner Alzheimer's Institute developed the GeneMatch program under the auspices of the Alzheimer's Prevention Registry (NCT02564692). GeneMatch, which is only open in the US, performs APOE genotyping in cognitively unimpaired individuals ages $55-75$ years in order to enrich referrals to prevention studies. Buccal swabs are sent to participants' homes or distributed at partner sites after online enrollment and consent. Although GeneMatch does not disclose APOE results to participants directly or inadvertently through invitations to studies, recruiting studies may require individuals to learn their results. As of August 2017, over 42,000 have joined GeneMatch, of whom nearly $4 \%$ are APOE4 HMs and 30\% are HTs; more than 900 have been referred to Generation Study 1.

\section{Conclusion}

Current therapies used in clinical practice for $\mathrm{AD}$ treatment aim to temporarily relieve symptoms, but they do not treat the underlying pathology of the disease. Advances in recent research have highlighted the importance of molecular processes in AD pathogenesis, which support the development of potential diseasemodifying agents. The most advanced pharmacological therapeutics for $\mathrm{AD}$ are anti-A $\beta$ monoclonal antibodies and BACE inhibitors; however, recent clinical failures of anti-A $\beta$ therapies in mild-to-moderate $\mathrm{AD}$ suggest that $A \beta$-directed treatments may need to be used earlier in the clinical course of the disease (11). Results from amyloid-based studies in preclinical models and early clinical programs support the premise that intervention is more effective in early stages of amyloid accumulation. Further, the recently defined diagnostic criteria of different preclinical stages of AD may help to identify an at-risk population with biomarker evidence of $\mathrm{AD}$ pathology or carrying a genetic risk (i.e. mutations in the amyloid pathway or carriers of APOE4 allele).

Importantly, the definitive studies to determine whether treatment in the preclinical stages will prevent cognitive decline are likely to take more than a decade to fully accomplish, highlighting the importance of scientific partnerships to drive these complex prevention studies. The Generation Program provides access to promising and suitable investigational treatments for individuals with the highest risk for developing AD. They are also designed to further develop the biomarker endpoints needed to evaluate preventative AD treatments, and will provide more critical evidence to support the amyloid hypothesis than clinical trials in patients with symptomatic AD. They will also provide a public resource of data and biological samples to help advance the scientific study and prevention of $\mathrm{AD}$ and help the field to find effective prevention therapies as quickly as possible.

Funding: This Generation Program is supported by Novartis Pharma AG, Basel, Switzerland and Amgen, Thousand Oaks, CA, USA, in collaboration with the Banner Alzheimer's Institute, Phoenix, AZ, USA. Generation Study 1 is supported by funding from the National Institute on Aging, part of the National Institutes of Health, as well as the Alzheimer's Association, FBRI, GHR Foundation and Banner Alzheimer's Foundation.

Acknowledgments: All authors were involved in preparing, reviewing, and approving this manuscript. The authors thank Jackie L. Johnson, PhD of Novartis Ireland Ltd for providing medical writing support/editorial support, which was funded by Novartis Pharma AG, Basel, Switzerland in accordance with Good Publication Practice (GPP3) guidelines (http:/ / www.ismpp.org/gpp3).

Conflict of Interest Disclosure: Cristina Lopez Lopez, Anglika Caputo, Fonda Liu, Marie-Emmanuelle Riviere, Marie-Laure Rouzade Dominguez, and Ana Graf: full or former employees of, and shareholders in Novartis Pharma AG. Cristina Lopez Lopez has a patent (PCT/IB2017/054307; licensed to Amgen). Ronald G. Thomas: research support from National Institutes of Health (U01 AG010483); consultant fees from Toyama. Jessica B. Langbaum: research support from National Institute on Aging (RF1 AG041705-01A1, UF1 AG046150), Genentech/ Roche, Novartis, Amgen; Banner Alzheimer's Foundation, FBRI, GHR, Nomis Foundation, Alzheimer's Association; consulting fees from Biogen, Lilly. Rob Lenz: employee and shareholder of Amgen. Eric M. Reiman: research support from National Institute on Aging (RF1 AG041705-01A1, UF1 AG046150, R01 AG031581, R01 AG055444, P30 AG19610), Novartis, Amgen, Banner Alzheimer's Foundation, Alzheimer's Association, GHR Foundation, F-Prime Biosciences Research Initiative and NOMIS Foundation; consultant fees from Alkahest, Alzheon, Axovant, Biogen, Denali, Pfizer, United Neuroscience, Zinfandel Pharma; patent to Banner Health (US Patent No. 9,492,114). Pierre Tariot: grants from Novartis, Amgen, Banner Alzheimer's Foundation, National Institute of Health, National Institute of Aging, Alzheimer's Associations, Genentech Roche; research support from National Institute on Aging, Arizona Department of Health Services, Alzheimer's Association, Banner Alzheimer's Foundation, FBRI, GHR, Nomis Foundation, Flinn Foundation, Geoffrey Beene Foundation: consultant fees from Acadia, Abbott Laboratories, AbbVie, AC Immune, Auspex, Boehringer-Ingelheim, Brain Test Inc., California Pacific Medical Center, Chase Pharmaceuticals, CME Inc., GliaCure, Insys Therapeutics, Pfizer, T3D; consulting 
fees and research support from AstraZeneca, Avanir, Lilly, Lundbeck, Merck \& Co., Roche, Takeda; research support only from Amgen, Avid, Biogen, Elan, Functional Neuromodulation (f(nm)), GE, Genentech, Novartis, Targacept; stock options in Adamas Pharmaceuticals.

Ethical standards: The studies described in this manuscript are conducted in accordance with ICH-Good Clinical Practice guidelines and the Declaration of Helsinki and are approved by the appropriate institutional review committees and regulatory agencies. Written informed consent is obtained from each participant before any study procedures.

\section{References}

1. Sperling RA, Aisen PS, Beckett LA, Bennett DA, Craft S, Fagan AM, et al. Toward defining the preclinical stages of Alzheimer's disease: recommendations from the National Institute on Aging-Alzheimer's Association workgroups on diagnostic guidelines for Alzheimer's disease. Alzheimers Dement. 2011;7(3):280-92.

2. Brookmeyer R, Gray S, Kawas C. Projections of Alzheimer's disease in the United States and the public health impact of delaying disease onset. Am J Public Health. 1998;88(9):1337-42.

3. Reiman EM, Langbaum JB, Fleisher AS, Caselli RJ, Chen K, Ayutyanont N, et al. Alzheimer's Prevention Initiative: a plan to accelerate the evaluation of presymptomatic treatments. J Alzheimers Dis. 2011;26 Suppl 3:321-9.

4. Bonham LW, Geier EG, Fan CC, Leong JK, Besser L, Kukull WA, et al. Agedependent effects of APOE epsilon4 in preclinical Alzheimer's disease. Ann Clin Transl Neurol. 2016;3(9):668-77.

5. Jansen WJ, Ossenkoppele R, Knol DL, Tijms BM, Scheltens P, Verhey FR, et al. Prevalence of cerebral amyloid pathology in persons without dementia: a meta-analysis. JAMA. 2015;313(19):1924-38.
6. Liu CC, Liu CC, Kanekiyo T, Xu H, Bu G. Apolipoprotein E and Alzheimer disease: risk, mechanisms and therapy. Nat Rev Neurol. 2013;9(2):106-18.

7. Reiman EM, Caselli RJ, Yun LS, Chen K, Bandy D, Minoshima S, et al. Preclinical evidence of Alzheimer's disease in persons homozygous for the epsilon 4 allele for apolipoprotein E. N Engl J Med. 1996;334(12):752-8.

8. Reiman EM, Chen K, Liu X, Bandy D, Yu M, Lee W, et al. Fibrillar amyloidbeta burden in cognitively normal people at 3 levels of genetic risk for Alzheimer's disease. Proc Natl Acad Sci U S A. 2009;106(16):6820-5.

9. Reiman EM, Langbaum JB, Tariot PN, Lopera F, Bateman RJ, Morris JC, et al. CAP--advancing the evaluation of preclinical Alzheimer disease treatments. Nat Rev Neurol. 2016;12(1):56-61.

10. Agency European Medicines Agency (EMA). Draft guideline on the clinical investigation of medicines for the treatment of Alzheimer's disease and other dementias. 2016.

11. Evin G. Future Therapeutics in Alzheimer's Disease: Development Status of BACE Inhibitors. BioDrugs. 2016;30(3):173-94.

12. Corder EH, Saunders AM, Strittmatter WJ, Schmechel DE, Gaskell PC, Small GW, et al. Gene dose of apolipoprotein E type 4 allele and the risk of Alzheimer's disease in late onset families. Science. 1993;261(5123):921-3.

13. Qian J, Wolters FJ, Beiser A, Haan M, Ikram MA, Karlawish J, et al. APOErelated risk of mild cognitive impairment and dementia for prevention trials: An analysis of four cohorts. PLoS Med. 2017;14(3):e1002254.

14. Ward A, Crean S, Mercaldi CJ, Collins JM, Boyd D, Cook MN, et al Prevalence of apolipoprotein E4 genotype and homozygotes (APOE e4/4) among patients diagnosed with Alzheimer's disease: a systematic review and meta-analysis. Neuroepidemiology. 2012;38(1):1-17. 